\title{
Precision Nephrology Is a Non-Negligible State of Mind in Clinical Research: Remember the Past to Face the Future
}

\author{
Michele Provenzano $^{a}$ Luca De Nicola $^{b}$ Michelle J. Pena ${ }^{c}$ Giulia Capitoli $^{d}$ \\ Carlo Garofalo $^{\text {b }}$ Silvio Borrelli ${ }^{\text {b }}$ Ida Gagliardi ${ }^{a}$ Laura Antolini ${ }^{d}$ \\ Michele Andreucci ${ }^{\mathrm{a}}$
}

${ }^{a}$ Renal Unit, Department of Health Sciences, "Magna Graecia” University, Catanzaro, Italy; ${ }^{\text {b} R e n a l ~ U n i t, ~ D e p a r t m e n t ~}$ of Advanced Medical and Surgical Sciences, University of Campania "Luigi Vanvitelli," Naples, Italy; 'Department of Clinical Pharmacy and Pharmacology, University Medical Center Groningen, Groningen, The Netherlands; ${ }^{\mathrm{d} C e n t e r}$ of Biostatistics for Clinical Epidemiology, School of Medicine and Surgery, University of Milano-Bicocca, Monza, Italy

\section{Keywords}

Precision nephrology · Chronic kidney disease ·

Personalized medicine · End-stage kidney disease ·

Cardiovascular disease

\begin{abstract}
CKD is a major public health problem. It is characterized by a multitude of risk factors that, when aggregated, can strongly modify outcome. While major risk factors, namely, albuminuria and low estimated glomerular filtration rate (eGFR) have been well analyzed, a large variability in disease progression still remains. This happens because (1) the weight of each risk factor varies between populations (general population or CKD cohort), countries, and single individuals and (2) response to nephroprotective drugs is so heterogeneous that a non-negligible part of patients maintains a high cardiorenal risk despite optimal treatment. Precision nephrology aims at individualizing cardiorenal prognosis and therapy. The purpose of this review is to focus on the risk stratification in different areas, such as clinical practice, population
\end{abstract}

research, and interventional trials, and to describe the strategies used in observational or experimental studies to afford individual-level evidence. The future of precision nephrology is also addressed. Observational studies can in fact provide more adequate findings by collecting more information on risk factors and building risk prediction models that can be applied to each individual in a reliable fashion. Similarly, new clinical trial designs can reduce the individual variability in response to treatment and improve individual outcomes.

(c) 2020 S. Karger AG, Basel

\section{The Complexity of CKD}

CKD is defined as the presence of kidney damage, albuminuria in primis, with or without estimated glomerular filtration rate $(\mathrm{eGFR})<60 \mathrm{~mL} / \mathrm{min} / 1.73 \mathrm{~m}^{2}$ for at least 3 months [1]. Incidence and prevalence of CKD have almost doubled in the last 3 decades, and the related CKDspecific mortality has ranked in the top 15 of the causes of death worldwide [2]. Owing to the new perspective of

$\begin{aligned} & \text { karger@karger.com } \\ & \text { www.karger.com/nef }\end{aligned}$
Karger ${ }^{\prime /}$




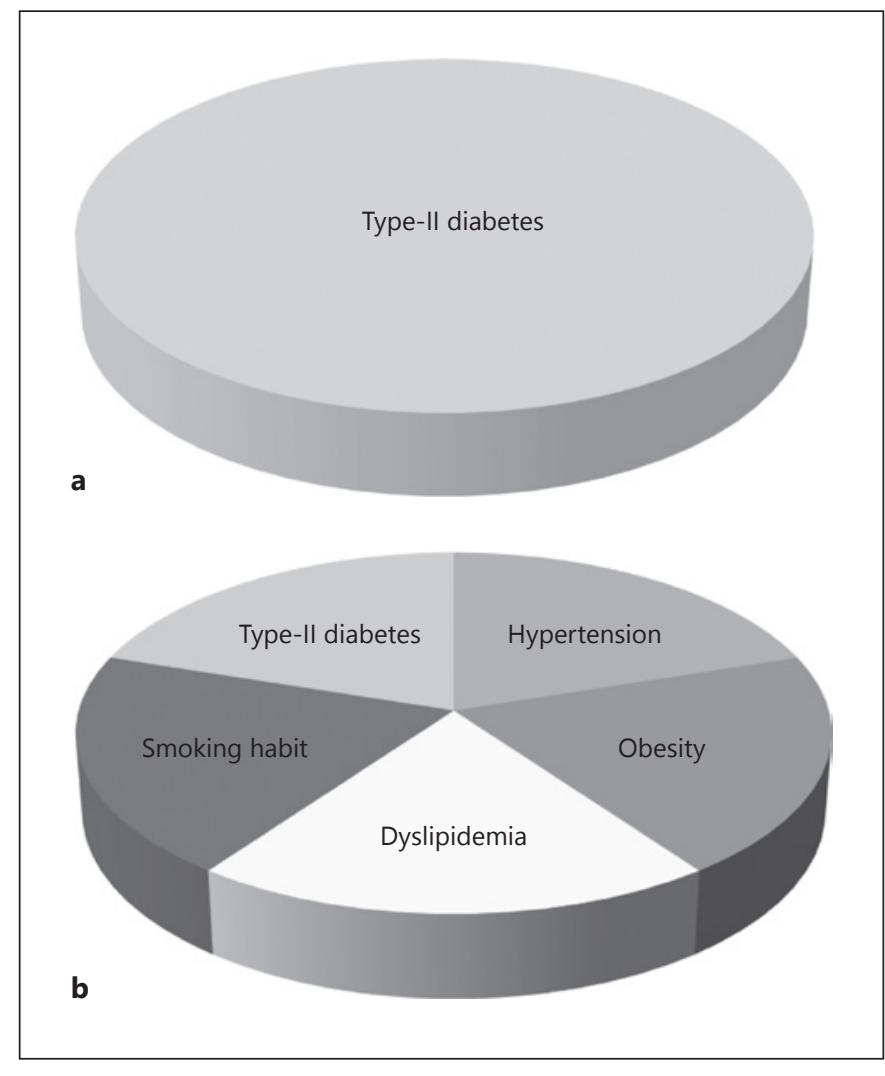

Fig. 1. Sufficient causes models in CKD: type II-diabetes alone as a cause of CKD (a), type-II diabetes may cause CKD only in the presence of other risk factors (b).

$\mathrm{CKD}$ as a global and public health problem, great effort has been made to identify patients at increased risk of developing unfavorable events over time. Indeed, predicting outcomes in CKD is crucial for clinical, public health, and research perspectives. In clinical practice, a correct prediction could help establish screening and treatment based on the individual risk profile; at the population level, predicting outcomes may guide the appropriate allocation of resources [3-6]. From the research perspective, the knowledge of specific outcomes is extremely advantageous for designing future clinical trials in terms of features of patients to be included, sample size dimension, end points to be measured, and duration of follow-up [7, $8]$.

Although a classification of CKD has already been established and spread to the nephrology community, one of the major problems that limits confidence of the clinicians in using prediction tools is that $\mathrm{CKD}$, as well as most chronic conditions, is characterized by a multitude of risk factors, which primarily influence the initiation and progression of the disease $[9,10]$. The pathophysiological network is complex because (1) several pathways cause CKD in some patients and not in others and (2) not all pathways that drive a specific injury are active in all patients at all stages [7]. We can simplify the concept using the causal-pie model, an instrument widely used in epidemiology, in order to represent when and why risk factors can play a role in the disease [11]. In the causal-pie models, a sufficient cause is the minimum set of factors that, if present in a given individual, will produce the disease. In the context of CKD, for example, it has been demonstrated that type 2-diabetes mellitus is the leading cause of ESKD [12]. However, although the presence of type 2 diabetes can per se be a sufficient cause for CKD onset (Fig. 1a), in many cases, development of kidney disease occurs only if other risk factors coexist (Fig. 1b). This epidemiologic concept is well supported by clinical studies. In the United Kingdom Prospective Diabetes Study, which enrolled about 5,100 patients with type 2 diabetes, only a fraction $(25 \%)$ of the population developed an overt nephropathy over a long-term (10 years) follow-up [13].

\section{Precision Nephrology}

Over the past centuries, clinicians have mainly used their proper medical knowledge, personal experience, and "intuition" to make decisions about the individual patient. Laboratory and clinical measures have been considered to make decisions on individual treatments (for instance, levels of blood pressure guided the decision to increase or reduce antihypertensive medications and hypercholesterolemia to introduce a lipid-lowering agent) and to predict future clinical outcomes as accurately as possible. These tasks represented a challenge for nephrologists and also gave fascination to this specialty. Precision nephrology is a branch of medicine that came forward with the noble aim of providing information and methodological tools that allow redefinition of CKD in terms of pathogenesis, prevention, prognosis, and treatment besides and beyond clinical intuition [14]. Precision nephrology includes many areas, including better phenotyping, improved elucidation of disease mechanisms, customization of medical decisions, and better risk stratification [15]. The major challenge for nephrologists and researchers, regarding precision nephrology, is ameliorating the knowledge of the mechanisms underlying the large variability of CKD. Variability can capture different meanings; with respect to prognosis, it can be translated into variability of patients' characteristics, disease progression, and frequency of outcomes. For treatment, variability refers to individual differences in response to treat- 
ment. These concepts appear to be extremely interconnected because reducing variability in drug response can reduce variability itself in disease progression, which in turn would allow a better prognosis to be obtained for each patient. Hence, precision nephrology encompasses both observational and experimental studies by focusing on individual estimates. Although the concept of "precision" is not new in medicine, the development of large databases, novel methods for characterizing patients (such as biomarker, proteomics, genomics, and metabolomics assessments), and new computational techniques adopted to analyze these data have increased the overall interest toward this discipline [16].

\section{Precision Nephrology in Observational Studies}

\section{Improving Patient Stratification: The Role of Risk \\ Scores}

A number of observational studies in nephrology have stratified patients into clinically relevant risk categories [17-19]. The purpose of all risk prediction models is, in fact, to allocate a larger number of patients in the true risk category, which corresponds to a low-risk category for patients who did not develop an event, or in a high-risk category for those who underwent the event itself [20]. The current classification of CKD considers 2 main parameters used to categorize CKD patients, namely, eGFR and albuminuria [21]. Even if the evidence available on the link between low eGFR or high albuminuria and worse outcomes are robust, such classification ignores the complexity of renal diseases and related risk factors as well. This was also defined as the "Reductionist approach" and a recent commentary showed as most nephrologists are steadfast with this approach [22]. In fact, both albuminuria and eGFR have been shown to have limitations in estimating individual prognosis in CKD patients. The presence of albuminuria, despite being strongly associated with the development of cardiovascular (CV) and renal events over time, fails to characterize CKD patients because it is a marker of all-cause kidney damage rather than being specific to 1 single primary renal disease [1, 21]. Moreover, it has been widely demonstrated that increased albuminuria is a hallmark of systemic endothelial dysfunction, and this explains why it predicts CV events also in the general population (GP) $[14,18,23]$. In contrast, eGFR is considered the best index to estimate the overall kidney function [21]. However, a reduction in eGFR may imply either progression of an underlying disease or the onset of a superimposed and often reversible

Precision Nephrology in Observational and Intervention Studies problem (e.g., decreased renal perfusion caused by volume depletion), or being merely dependent on the biological and analytical variations in serum Cr levels. Similarly, an increase in eGFR may also not herald a good prognosis if it is the sign of hemodynamic hyperfiltration as is often seen in the early stage of diabetic kidney damage [24]. The use of eGFR and albuminuria, as criteria of CKD detection, leads to an overall late diagnosis of this condition, being rarely involved in the initiation of kidney disease [14]. In addition, how often albuminuria and eGFR should be measured over time and what changes of these 2 kidney measures predict cardiorenal risk accurately both remain to date clinically meaningful unanswered questions. Following the "reductionist" approach, for example, it is not clear how to incorporate the primary renal diagnosis of CKD into prognostic categories, despite the important finding that renal diagnosis plays a significant role in predicting mortality and CKD progression regardless of albuminuria and eGFR with a weight that is almost equal to that of albuminuria $[25,26]$.

In the era of precision medicine, the aim has been changed by focusing more on individual risk. An initial effort has been undertaken to incorporate risk factors of CKD into multivariable scoring systems that provide risk estimates for small group of subjects, rather than calculating an average risk for each variable or disease category [27]. Such a method is similar to the Framingham Risk Score that is currently used to determine the individual risk for ischemic heart disease in GP [28]. It is important to emphasize that the risk value for a single subject, computed through an individual prediction model, provides the risk of the group of subjects with the same predictors of the examined subject and not the probability of developing an event in a random patient. This permits to compute, for example, a 10-year risk (\%) of coronary heart disease in a 65-year-old man, current smoker, with 150 $\mathrm{mmHg}$ of systolic blood pressure and $149 \mathrm{mg} / \mathrm{dL}$ of LDL cholesterol. In this way, the estimate obtained for an individual risk model could be applied to new patients to predict outcome. This attractive approach can be considered a first step toward precision nephrology by using observational data. The main advantage of the risk scores is that they combine the prognostic information derived from multiple parameters, thus improving the accuracy in predicting individual patient outcome. Physicians would be, therefore, facilitated in deciding whether and how to intensify or reduce monitoring and treatment. The investigators of reduction of end points in NIDDM with the angiotensin II receptor antagonist losartan study framed the first score for patients with type 2 diabetes and 
nephropathy [29]. This score focused on the prediction of CKD progression, and ESKD onset and was, therefore, labeled as renal risk score. The authors demonstrated that, by combining the effects of urine albumin-to-Cr ratio (ACR), serum albumin, hemoglobin, and serum $\mathrm{Cr}$, prediction of ESKD improved by $30 \%$, as compared with serum Cr alone. In subsequent years, Tangri et al. [27, 30] developed a risk prediction model, validated internationally, the so-called Kidney Failure Risk Equation that accurately predicts the progression to ESKD for the individual patient with $\mathrm{CKD}$ stage III to $\mathrm{V}$ by input of age, gender, ACR, and eGFR. More recently, an additional score for estimating ESKD risk in CKD patients (stage 3 or 4) has been published by the Kaiser Permanente Northwest, a health maintenance organization that serves several areas of the US [31]. The CKD-Prognosis Consortium also developed and validated risk scores for the prediction of ESKD, nonfatal CV events and death in advanced CKD (eGFR $\left.<30 \mathrm{~mL} / \mathrm{min} / 1.73 \mathrm{~m}^{2}\right)$ [32, 33]. These prediction models may significantly improve both the understanding of prognosis of patients with CKD and change the perspective of the decision-making process in nephrology from the eGFR-based to the multimarkerbased approach [34].

However, the risk scores do not fully reach approval and diffusion within the nephrology community $[35,36]$. Nephrologists are in fact well aware that the CKD population has a complex nature, due to the large heterogeneity of patients and outcomes. Three strategies have been, therefore, proposed to improve the implementation of risk prediction models [36]. The first one is to consider the sources of variability of CKD in building the model as accurately as possible. The second one consists of improving the methodological development and validation of a prediction model. Indeed, although it may seem counterintuitive, an individual risk prediction model must satisfy many methodological steps before being transferred into clinical practice [37]. Intriguingly, a recent systematic review has shown that only a few studies, aimed at predicting the risk of CKD progression, did actually meet the criteria for clinical decision-making [38]. The third strategy, which is similarly critical, is to favor dissemination among nephrologists of risk prediction models.

\section{Sources of Variability in CKD}

Variability of CKD can be depicted over different scales. From a general view, CKD patients referred to outpatient nephrology care constitute a peculiar, high-risk, setting. Referred patients profoundly differ from the un- selected CKD GP in terms of basal characteristics and prognosis. Prevalence of type 2 diabetes reached $34-46 \%$ in the referred CKD patients of Chronic Renal Insufficiency Cohort and Italian multicohort, respectively, whereas prevalence of established CV disease (CVD) was about $25-30 \%[39,40]$. CKD patients selected from the GP are characterized by a lower intrinsic risk profile compared with those who are referred with the prevalence of diabetes and CVD being often below 10-15\% [41]. As expected, these features have a major impact on prognosis. Indeed, absolute risks of ESKD, CV fatal and nonfatal events, and mortality are significantly higher in referred CKD patients compared with the GP. In the Italian multicohort, $21.4 \%$ of patients reached ESKD after an average of 4.1 years, whereas a much smaller proportion of subjects $(0.3 \%)$ developed the same end point over the same time frame in the Alberta Kidney Disease Network that was built with the aim of identifying patients at high risk for CKD in the Canadian province of Alberta [42, 43].

Variability is also present with respect to CKD prognosis. Many longitudinal studies, which measured the eGFR decline over time, reported different values in terms of punctual estimates and measures of variability. In a joint analysis of CKD cohorts from Europe, eGFR decline ranged from $0.77 \mathrm{~mL} / \mathrm{min} /$ year in the Belgium cohort to $2.43 \mathrm{~mL} / \mathrm{min}$ per year in the Spanish cohort [44]. Moreover, in a cohort of referred CKD patients, the median change of eGFR over 2 years was $-1.7 \mathrm{~mL} / \mathrm{min} / 1.73 \mathrm{~m}^{2} /$ year with an interquartile range of -4.6 to $+0.8 \mathrm{~mL} /$ $\min / 1.73 \mathrm{~m}^{2} /$ year [45], thus indicating substantial heterogeneity. Overall, the nonlinear eGFR trajectories, defined by the presence of periods of rapid declines alternating with periods of stability, may occur in up to $42 \%$ of CKD patients $[46,47]$. Noteworthy, this large variability of disease progression has also emerged in randomized clinical trials despite the strict selection of high risk patients (presence of high albuminuria and/or low eGFR levels). An example is the placebo group of the Irbesartan Diabetic Nephropathy Trial, where eGFR decreased by $6.5 \mathrm{~mL} / \mathrm{min} / 1.73 \mathrm{~m}^{2}$ per year with a coefficient of variation (standard deviation/mean) of 135\% [48].

Variability can also emerge between geographic areas. The association between CV risk factors and mortality is different between the US and Europe, the age-adjusted mortality for coronary heart disease being 3 -fold higher in men from the US and Northern Europe than Mediterranean Europe, even when similar values of blood pressure and cholesterol are presented [49]. This is in accordance with the different degree of inflammatory status in CKD patients of the US and Europe [50]. 


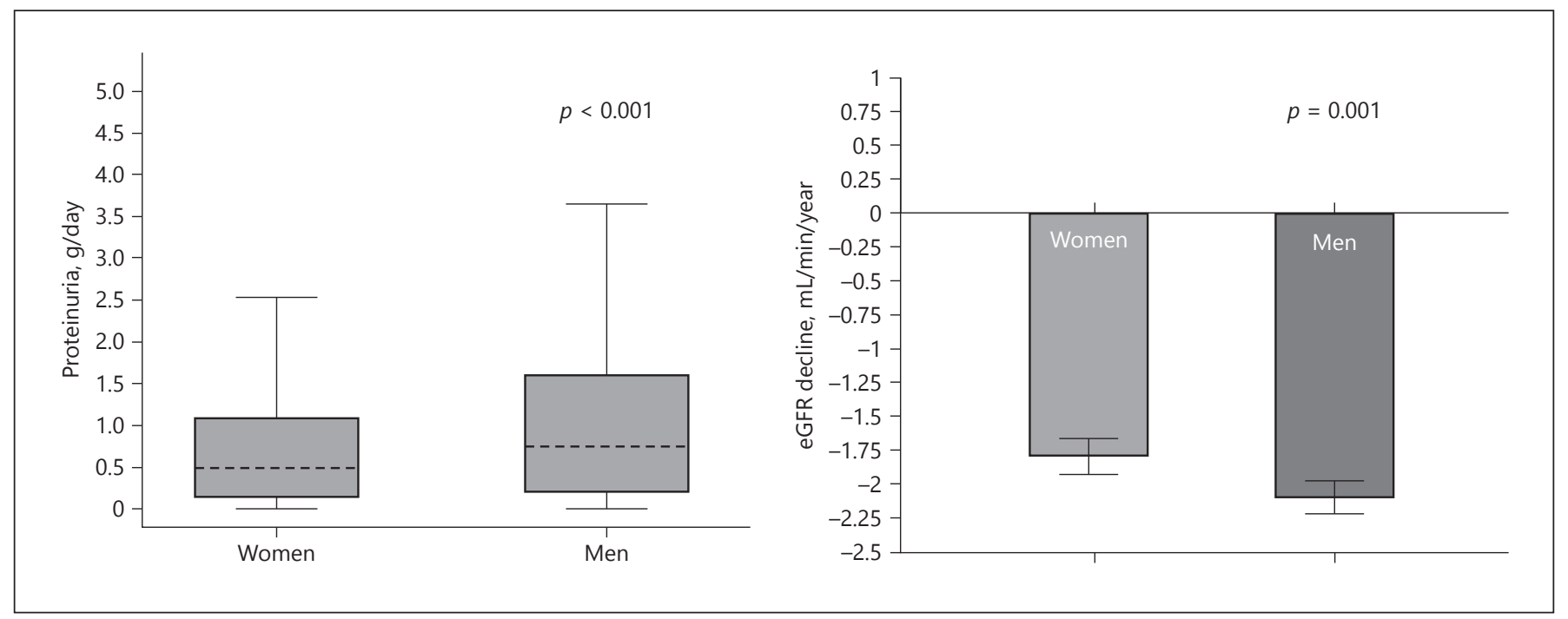

Fig. 2. Gender differences in 24 -h proteinuria levels (left panel) and eGFR decline (right panel) in a cohort of 2,335 CKD patients referred to nephrologists [53]. eGFR, estimated glomerular filtration rate.

In the past years, numerous prognostic models have shown that besides eGFR and albuminuria, numerous covariates, such as age, gender, presence of diabetes or metabolic syndrome, primary renal disease, blood pressure, hemoglobin, previous CVD, and serum phosphate, are strong predictors of eGFR decline, ESKD, CV events, and mortality $[10,17,18,25,27]$. Nevertheless, the role and the weight of each risk factor for disease progression are still unknown. For example, even if albuminuria acts a toxic effect on the glomerulus and the renal tubule, many patients progress without or with a low degree of albuminuria [6]. Similarly, a consistent number of diabetic patients remain at increased risk of CKD progression and mortality despite a non-albuminuric phenotype [51].

Among demographic and clinical variables that are sources of individual variability, it is interesting to mention the variability related to sex. It has been shown that men seem to have a faster eGFR decline than women [52]. More recently, Minutolo and colleagues have reported how this excess of renal risk in men is partially associated with a dissimilar burden of proteinuria between men and women (Fig. 2) [53]. This impressive finding can be translated into the practical concept that nephrologists should differentiate monitoring also by the sex of patients.

All these sources of variation are potentially sources of bias in building a prediction model. This concept has probably contributed to the limited dissemination of prediction models in nephrology practice.

Precision Nephrology in Observational and Intervention Studies
Framework for Developing Individual Risk Prediction Models for Use in Precision Nephrology

To implement risk scores into clinical practice, it is recommended, according to previously published literature, to tackle several methodological steps. The first step consists of clarifying the purpose, or the "intended use" of the model. Patients want to know their specific risk and if the prediction is reliable. On the other hand, the aim of clinicians is to predict an outcome in a way that outperforms their clinical intuition or ability. Hence, the intended use should include the population evaluated, the predicted outcomes, and the variables used to compute prediction. The second step is selecting population from which the risk prediction model will be derived. It is highly plausible that a risk score built in CKD patients provides different results from another similar score derived from the GP or from a high-risk population other than CKD. Hence, a sufficient number of patients with different degrees of kidney impairment as well as different primary renal diagnoses should be included and several basal characteristics need to be considered. In the case of too many variables included, stratified models and interaction analyses are available to better assess risk stratification and reach a proper "phenotyping" of CKD [54]. The third step is the outcome selection. It is noteworthy that all available risk scores have focused on renal end points, such as ESKD and CKD progression. Recently, a validation of the Kidney Failure Risk Equation has been extended to elderly CKD patients for the prediction of mortality end point 


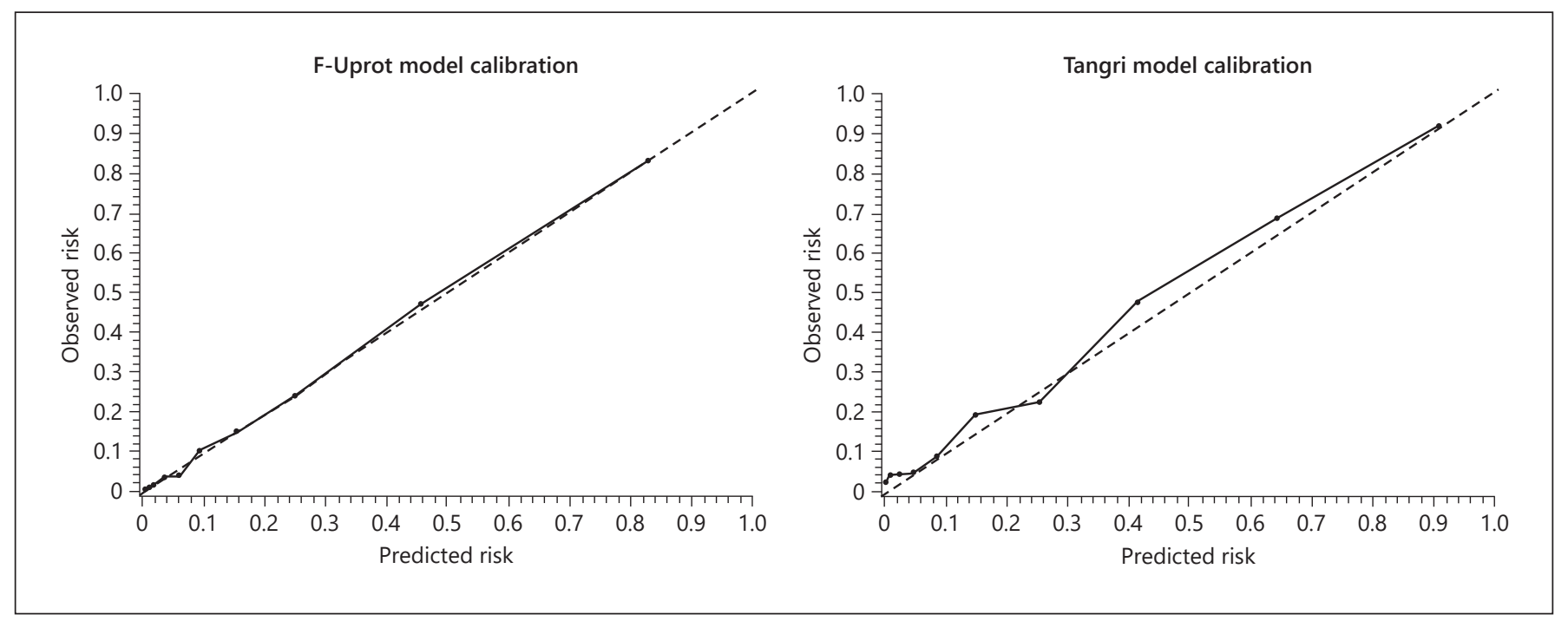

Fig. 3. Calibration plot of predicted versus observed ESKD risks derived from 2 multivariable prediction models in a cohort of 3.957 CKD patients in Italy (Tangri model calibration, model with proteinuria, age, gender, eGFR; F-Uprot model, Tangri model with proteinuria replaced by proteinuria indexed to eGFR) [40]. eGFR, estimated glomerular filtration rate.

[55]. However, a CV risk score, predicting fatal and nonfatal CV events in CKD population, is still highly awaited, particularly after the description of the lack of accuracy of Framingham risk score when applied to CKD patients [56]. The perspective of CKD as CV risk equivalent has, indeed, raised concerns in the scientific community because, following this statement, all patients with CKD are now considered at high risk for CV events, regardless of the exact value of eGFR, albuminuria, smoking habit, cholesterol levels, blood pressure, and several other parameters, whose weight needs to be assessed [57]. Potential other outcomes of interest are acute kidney injury, stability of eGFR over time, and hospitalizations [31, 58].

The next step to be included in the model is selecting variables. Individual risk prediction models are generally represented by multivariable models. Unlike the associative models, for which the aim is to evaluate the relationship between exposure and the clinical outcome, the goal of individual risk prediction models is to generate the best possible estimate of the value of the outcome variable for each subject. The focus of these models is, therefore, the outcome rather than the predictors. Variables selection could be done with data-driven automated methods or based on the plausible (already known) biological link between exposure and outcome (knowledge-driven or "a priori" selection). Either method is acceptable and widely used in the literature, while the decision of what is more appropriate depends on the research question [59].
Use of a rigorous methodology is essential. In order to provide appropriately individual risk estimates, it is not sufficient to build and present a multivariable model. Irrespective of the aim, the first question to be answered is whether the model is "reliable" and can be used in clinical practice. The statistical tool that provides this measure is calibration. Calibration is the degree of agreement between observed and predicted outcomes. It is usually shown graphically by plotting the mean predicted probability versus the mean observed outcome for intervals of risk (deciles) in a predictiveness curve or by plotting observed event rates versus average modeled risk, thus producing points that should lie along a $45^{\circ}$ line if the model is well calibrated. This is called the calibration plot [37]. Figure 3 represents an example of a calibration plot computed on a cohort of 4,000 CKD patients referred to nephrology clinics in Italy [40]. In support of graphical assessment, the Hosmer-Lemeshow test is often presented as test of calibration, based on the comparison between observed and predicted risks. Even if calibration is assessed on the dataset for which the model was developed, it may not be valid in all study populations. Beyond calibration, it is central, from a statistical perspective, to quantify overall model performance, the so called "goodness of fit" (GOF), describing how well the model fits a set of observations. In order to compute GOF, the most frequently used measures are the explained variation of the model $\left(R^{2}\right)$ and the Brier score. $R^{2}$ expresses the per- 
Table 1. Principal measures for assessing performance of risk prediction models

\begin{tabular}{|c|c|c|}
\hline Statistical metrics & Description & Measures \\
\hline Calibration $[36,37,57]$ & $\begin{array}{l}\text { Measure of the degree of correspondence between } \\
\text { observed and predicted risk. Miscalibrated model } \\
\text { produces invalid risk estimates and can introduce } \\
\text { errors in decision-making. }\end{array}$ & $\begin{array}{l}\text { Graphical plots } \\
\text { - predictiveness curve } \\
\text { - calibration plot } \\
\text { Tests } \\
\text { - Hosmer-Lemeshow }\end{array}$ \\
\hline Goodness-of-fit $[36,57,59]$ & Measure that quantifies the overall model fit & $\begin{array}{l}\text { Brier Score; } \\
\text { Nagelkerke's R }{ }^{2}\end{array}$ \\
\hline Reclassification [36] & $\begin{array}{l}\text { Measure of the movement of patients from one class } \\
\text { to another based on changes to assignment to risk } \\
\text { categories. }\end{array}$ & $\begin{array}{l}\text { Continuous NRI (Net Reclassification } \\
\text { Improvement) } \\
\text { Categorical NRI } \\
\text { Integrated discrimination improvement (IDI) }\end{array}$ \\
\hline
\end{tabular}

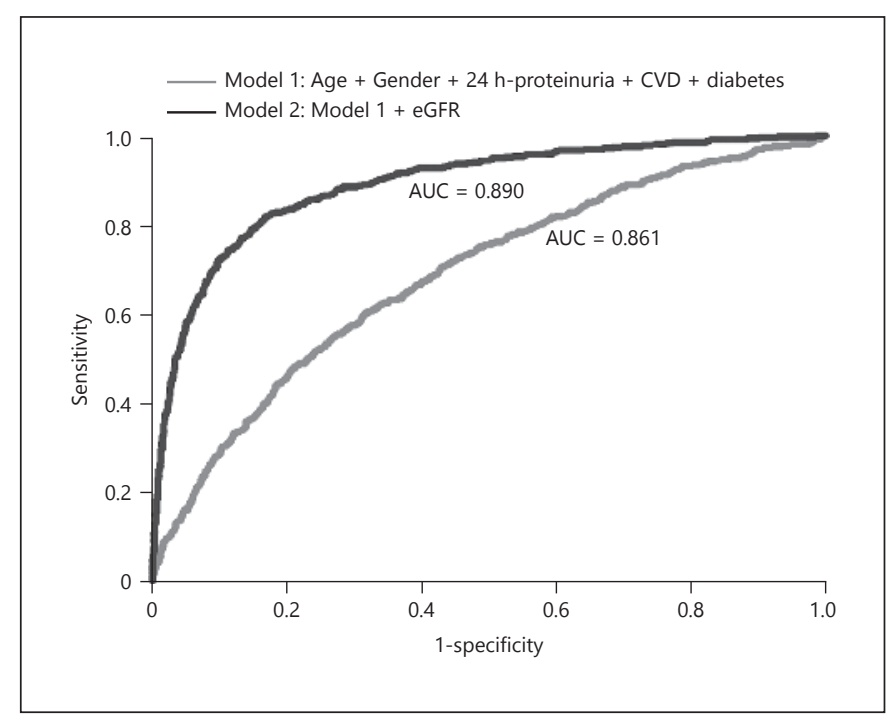

Fig. 4. ROC curves for the prediction of ESKD event within 3 years of follow-up. CVD, previous cardiovascular disease; eGFR, estimated glomerular filtration rate; ROC, receiver operating characteristic; AUC, area under the curve.

centage (\%) of contribution of a covariate of interest to the overall model-explained variation, thus allowing the estimation of the weight of each risk factor [60]. The Brier score computes the squared difference between observed outcomes and predictions [61]. A further step, discrimination, is required. Discrimination evaluates the

Precision Nephrology in Observational and Intervention Studies separation between distribution of the risk predictor in events and in nonevents. A prediction model for a binary end point produces a risk score depicting the predicted probability of experiencing an outcome given the observed values of a set of covariates. Therefore, discrimination will show whether patients, who develop the event, have higher risk predictions than those who do not. Discrimination is tested by computing receiver operating characteristic (ROC) curve that plots sensitivity and specificity for the entire range of possible classification thresholds. A common way to summarize the ROC curve is the area under the curve, also known as $c$ statistic. That parameter measures the probability that the individual risk is higher for the end point-positive than the end pointnegative patients. Both ROC curve and $c$ statistic can be calculated from survival models. Figure 4 shows the ROC curve evaluating the discrimination ability of 2 prediction models, with and without eGFR, for ESKD risk within 3 -years of follow-up. More complex reclassification metrics, such as net reclassification improvement (NRI) and integrate discrimination index (IDI), are available when the research question is to assess the incremental value of a biomarker on top of traditional, already well-accepted risk factors (Table 1). NRI can be calculated by computing the difference between the proportions of individuals moving up and the proportion of individuals moving down for those who develop end points and the corresponding difference in proportions for those who not de- 
velop end points. The IDI measures the differences in average risk probabilities between events and nonevents. This parameter is considered more accurate than NRI because, unlike the NRI, IDI is independent from risk categories and estimates the magnitude of reclassification.

The final step of each prediction model is to guarantee its transportability, the ability of the model to provide accurate predictions in a different sample of patients. The best way to assess transportability is the validation in a fully independent, external data, the so-called external validation. When an external cohort is not directly available, it is possible to test the internal validation. However, internal validation is useful to assess the reproducibility (in a different cohort with similar characteristics) and can be computed using bootstrapping or cross-validation methods [61].

\section{Improving Development and Diffusion of Risk}

Prediction Models to the Nephrology Community

The risk prediction tools have been widely implemented in CV medicine [62]. The European guidelines on CVD prevention supported the use of the individual risk scores for predicting CV outcomes and for individualizing preventive and therapeutic strategies [63]. Several initiatives have also been started to stimulate the dissemination of these tools. The Joint British Societies and the Framingham Investigators created an online tool through which individuals can identify and select the risk factors that need to be changed. Such self-assessed scores helped individuals to gain awareness about the underlying disease and the benefits of behavioral or prevention changes. Moreover, support to clinicians or patients for selecting the best prediction tools for each individual based on medical history, outcome measured, and methodological validation has also been provided [64]. Conversely, in the context of nephrology, the routine application of risk scores is still lacking, and this is determined by several cultural or practical obstacles [36]. Clinicians are usually uncertain about the discrepancy between the simplicity of risk prediction scores and the clinical complexity. In CKD patients, this is complicated by the fact that prognosis is multifactorial and heterogeneous and clinical outcomes need several years, even decades, to manifest. Another nontrivial limitation is that in the nephrology field, marketing and branding of new concepts and tools are not as easy as in other fields of medicine. The mentioned technological steps, already taken in the CV field, have been invoked also for the nephrology community. In 2016, the International Society of Nephrology (ISN) planned a set of activities, called "closing the gaps," aimed at improving the global outcome of CKD patients [65]. ISN enhanced, with this long-time project, the need to cooperate with other professional organizations and regulatory agencies, such as the National Kidney Foundation and the Food and Drug Administration (FDA) to realize large-scale prediction models, which include kidney measures, and to incorporate the final results in the international guidelines. Moreover, other objectives of the ISN initiative are (1) to involve patients actively in the clinical decision-making, as this patient-centered approach could be useful for achieving better health outcomes and patient satisfaction; (2) to build up training for young researchers focused on the development, validation, and clinical application of risk prediction models [66]. What is certainly needed in nephrology is the development and validation of risk models that could demonstrate an improvement in the management of CKD when compared with the standard clinical care. This step goes along with the assessment of the role of new biomarkers in CKD. A great effort has been made in the past decades to find novel biomarkers which may improve "accuracy" of already existent risk prediction models. The use of cystatin $C$ for estimating eGFR has shown to improve the discrimination of patients with and without CKD and also to refine CV and renal risk prediction $[67,68]$. Blood levels of cardiac troponins (high sensitivity cardiac troponin [hs-cTnT]) and natriuretic peptides ( $\mathrm{N}$-terminal pro-B-type natriuretic peptide [NT-proBNP]) have both been associated with the development of heart failure and ESKD in patients with CKD, regardless of traditional risk factors and kidney measures (eGFR and proteinuria) [67]. Increased levels of fibroblast growth factor 23 appeared to predict worse end points in CKD patients as well [69]. To account for the large heterogeneity of $\mathrm{CKD}$, more sophisticated approaches for biomarker detection have also been used [70]. Proteomics provided an important contribution in finding biomarkers from blood, urine, and kidney biopsies [71]. Furthermore, proteomics allowed combining the information obtained from panels of peptides to derive a combination of these peptides that classifies patients - hence the name of classifier - into relevant clinical or risk categories. A promising classifier is CKD273, a panel of 273 urine peptides that was primarily found to detect CKD risk regardless of the underlying etiology [72]. Intriguingly, in following cohort studies with up to 7 years of follow-up, CKD273 was able to predict eGFR decline in CKD patients (with multiple etiologies) beyond eGFR and proteinuria, even at early stages of kidney disease $[70,73,74]$. More specifically in diabetic patients, CKD273 has been shown to predict the onset of albumin- 
uria and the progression to diabetic nephropathy with a higher accuracy when compared with the 2 kidney measures (eGFR and albuminuria) alone [75]. However, the additional value of measuring a biomarker/classifier includes the fact that it refines risk stratification, but also that is able to identify therapeutic targets for intervention (biomarker guided treatment). This means that each significant change of the biomarker level in response to a treatment must be associated with a change in the risk of developing an unfavorable outcome. Such implementations overall need reliable threshold tests to be performed and that studies comparing biomarker guided treatment versus standard of care should be kicked-off. All these strategies could also represent a positive way to close the gap also with respect to other fields of medicine and to increase the social awareness of CKD, which is still low; indeed, only $10 \%$ of patients are aware of their future risks [41].

\section{Precision Nephrology in Intervention Studies: Reducing Variability in Drug Response to Improve Patients' Prognosis}

\section{Variability in Drug Response}

Along with the increase in observational cohort studies, many intervention studies have been carried out in the past 2 decades with the final goal of achieving a better control of CV and renal risk in CKD patients [76-78]. Drugs that inhibit the renin-angiotensin-aldosterone system (RAAS-I) reduce CV and renal risk [79, 80]. Other studies have assessed the effect of other agents like erythropoiesis-stimulating agent in the TREAT trial, endothelin receptor antagonist in the SONAR trial, and inhibitor of sodium-glucose cotransporter 2 in the CREDENCE trial [81-83]. Although all these trials have revealed the possibility to reduce the ESKD and CV risk, a large portion of the trial population was left at high residual risk despite optimal treatment. Moreover, the presence of CKD is per se an additional risk factor that contributes to the residual risk of CV events [84]. A major reason why these multiple interventions do not confer a full risk reduction is the individual variability in drug response. Several analyses of past clinical trials have examined the frequency and the determinants of individual response to treatments. In some albuminuria end point studies, patients who respond in the first months, in comparison with those who do not respond at all or who respond later, show the greatest risk reduction of developing hard end points in the long term $[85,86]$. Moreover, the vari-

Precision Nephrology in Observational and Intervention Studies ability in albuminuria response is reproducible upon reexposure to a drug, when an anti-albuminuric drug is uptitrated or when another drug of the same class is administered [87, 88].

The second reason, which further complicates all these mechanisms, is that nephroprotective drugs act by changing several biomarkers other than those usually measured to assess their own efficacy, which is the "primary" target. Several drugs determine "secondary" effects; typical examples are the effects of RAAS-I on hemoglobin, serum potassium, blood pressure, and uric acid levels or the effect of endothelin receptor antagonist on body weight. The sum of all these effects, rather than the single primary response, determines the final patients' outcome. Intriguingly, it has also been demonstrated that a certain degree of variability is present also for the "secondary" effects of each drug [89]. The importance of such findings has further raised the interest in evaluating how to overcome individual therapy resistance and reduce this wide variability in disease progression as much as possible. A lower variability in response to treatment may lead to a lower variability in CKD progression, better characterization of existing drugs, and disease pathways, which in turn should allow better prognosis. This point represents a major objective of precision nephrology. In fact, the variability in response to treatment affects not only the drug development but also the individual patient care. It has been shown that in patients with diabetic kidney disease, the residual risk is extremely high, being similar to the mortality in treated cancers [90]. In CKD patients from all etiologies, the residual risk for both CV fatal and nonfatal events and renal events was 2- to 3-fold higher in patients with a residual proteinuria of $0.5 \mathrm{~g} /$ day or higher, albeit under maximum RAAS inhibition [42]. To provide more insights into individual variability in drug response, the "ideal" path to follow is to find out the mechanism of damage in each individual and to find the drug that can significantly influence that mechanism, thus pursuing a targeted treatment approach. The progress obtained by "omics," namely, novel techniques used to analyze molecular processes associated with the disease (genomics, proteomics, metabolomics, and transcriptomics) will likely allow the discovery of novel pathways of damage and consequently potential targets for therapeutic interventions [71, 91]. Genome-wide association studies identified the variants of the UMOD gene that cause overexpression of uromodulin and upregulation of the tubular transporter NKCC2. The effects of the pharmacological inhibition of NKCC2 in lowering blood pressure strictly depend on expression of uromodulin, the lat- 
Table 2. Principal types of new clinical trials designs assessing the individual response to treatment

\begin{tabular}{|c|c|}
\hline Trial design & Description \\
\hline Biomarker-based enrichment $[7,90]$ & $\begin{array}{l}\text { Patients are selected on the basis of the presence of absence of a marker of disease. This } \\
\text { approach may also include patients at increased risk of developing a disease, thus allowing to } \\
\text { anticipate treatment. }\end{array}$ \\
\hline Adaptive enrichment [81] & $\begin{array}{l}\text { All patients who meet inclusion criteria start a run-in phase in which they receive the study } \\
\text { drug. Only patients who respond to the experimental treatment are then randomized to } \\
\text { placebo or control group. }\end{array}$ \\
\hline Multimarker-based enrichment [81] & $\begin{array}{l}\text { Patients are selected based on the individual response to many, primary and secondary, risk } \\
\text { markers. }\end{array}$ \\
\hline Cross-over design $[105,106]$ & $\begin{array}{l}\text { Patients are randomly assigned to receive a sequence of different treatments. These studies } \\
\text { uncover the information about which drug each patient shows the best response. }\end{array}$ \\
\hline n-of-1 design $[105,106]$ & $\begin{array}{l}\text { Also called single subject clinical trials, consider an individual patient as the sole unit of } \\
\text { observation in a study investigating the efficacy or side-effect profiles of different drugs. } \\
\text { The ultimate goal of these studies is to determine the optimal or best intervention for an } \\
\text { individual patient. }\end{array}$ \\
\hline Umbrella design [105] & $\begin{array}{l}\text { Testing multiple targeted therapies in a single disease where various biomarker-based } \\
\text { subgroups are identified and different drugs are tested in these subgroups of patients. }\end{array}$ \\
\hline Basket design [105] & $\begin{array}{l}\text { Testing a single targeted therapy in multiple diseases that could benefit from the same drug, } \\
\text { given the etiology and pathophysiological mechanisms. }\end{array}$ \\
\hline Platform design [105] & $\begin{array}{l}\text { Patients are constantly followed over time with laboratory and clinical exam (that are } \\
\text { recorded in a platform) and intervention drugs allowed to enter or leave on the basis of } \\
\text { precision schemes. This strategy allows to conduct clinical trials for personalized medicine } \\
\text { quickly and at lower costs. }\end{array}$ \\
\hline
\end{tabular}

ter, therefore, being a possible therapeutic target for preserving renal function by reducing blood pressure [92]. Similarly, the discovery that DNA hypermethylation was associated with the downregulation of the KLOTHO gene and with a worse prognosis of CKD identified KLOTHO as another potential target with the aim of slowing CKD progression [93]. Proteomics detected, other than the classifier CKD273, several other interesting biomarkers, such as the transforming growth factor beta 1 (TGF- $\beta 1$ ). TGF- $\beta 1$ plays an important role in the pathogenesis of glomerular and tubular fibrosis [94]. It has raised great interest, so that the development of drugs can directly neutralize that polypeptide [95].

However, the application of all these findings in the context of precision nephrology is not straightforward. What complicates this step is that CKD is multifactorial, with the pathways involved in initiation, progression, and comorbidities being complex and often different. One necessary way is to predict the individual response to a specific treatment by using biomarker panels (biomarker guided treatment). Such a strategy has been tested in diabetic kidney disease with the response panel pa- rameter response efficacy score, a combination of both clinical and laboratory parameters that have been used to predict the $\mathrm{CV}$ and renal outcomes, based on the change of the parameters included in the score (urinary ACR, systolic blood pressure, hemoglobin, uric acid, potassium, and cholesterol levels) in response to a treatment [96]. These panels, however, should be implemented by including all the other CKD etiologies and treatment effects [70]. Another promising method, already developed by Mayer et al. [97], is the use of molecular pathways or network models to obtain, for each patient, a specific molecular process associated with a specific phenotype of CKD and also biomarkers associated with these processes. By matching the mechanism of a drug's action with these molecular models, we could also be able to select a correct treatment as accurately as possible and to better depict the individual variability to drug response in the future.

\section{New Designs of Clinical Trials}

A great effort in designing new clinical trials (RCT) for reducing the variability in response to treatment and 
prognosis of $\mathrm{CKD}$ is ongoing. One key example is the novel approach to RCT design in diabetic kidney disease (Table 2) [7]. Three different strategies that use biomarkers have been recently proposed: biomarker-based enrichment approaches, individual response-enrichment designs, and multiple biomarkers-based enrichment.

The first approach, also called target RCT design, consists of enriching the population with patients at increased risk of disease progression. The PRIORITY trial (proteomic prediction and renin angiotensin aldosterone system inhibition prevention of early diabetic nephropathy in type 2 diabetic patients with normoalbuminuria) enrolled normoalbuminuric patients at higher risk of developing microalbuminuria [98]. This risk estimate has been derived from the urine proteomic classifier (CKD273) that was used to discriminate high- and low-risk patients. Only high-risk patients $(n=209$ out of 1,775 total participants) were randomized to receive spironolactone or placebo and the development of confirmed microalbuminuria was assessed as primary end point. High-risk patients, identified with the urinary CKD273, were at significantly higher risk of progression to microalbuminuria, compared with low-risk patients $(p<0.001)$, thus confirming the validity of the classifier as an early marker of risk of kidney disease progression in diabetic patients. However, spironolactone did not prevent the development of microalbuminuria in patients at high-risk of renal progression selected on the basis of CKD273 urinary levels $(p=0.41)$. Reasons to explain the lack of effect of spironolactone on the primary outcome have been principally related to the study power [99]. In fact, the number of patients allocated in the high-risk group was lower than the 1 planned in the sample size calculation (12 vs. 20\%) [98]. This finding, together with the low rate of event in the placebo group, reduced the study power. However, it could not be excluded that an absence of spironolactone effect in this population gambled a decisive role [98]. By contrast, it is important to emphasize that, in this study, originally, an anti-albuminuric drug is primarily started in patients without albuminuria, but at increased risk to develop it. This is an example of RCT design guided by a prognostic classifier that could be, in this case, informative for planning early intervention. Alternative albuminuria-lowering drugs, such as SGLT-2 inhibitors, should be tested in the future in patients at increased-risk of albuminuria detected by urinary proteomics [98].

An alternative approach is to enroll patients who are likely to respond to a new intervention (individual response-enrichment designs or, also called, adaptive en-

Precision Nephrology in Observational and Intervention Studies richment design). This type of enrichment approach was used in the study of diabetic nephropathy with the endothelin receptor antagonist atrasentan (SONAR). An enrichment period was performed to separate atrasentan responders from nonresponders based on the level of reduction in urinary ACR. All patients who qualified for the trial at screening were exposed to 6 weeks of atrasentan therapy. Then, only patients who responded to atrasentan, defined by a $30 \%$ reduction in UACR, were randomized into placebo or active arms. Importantly, the SONAR trial can also be considered a multiple biomarkers-based enrichment RCT. Indeed, it also considers the individual's response in "secondary" risk markers (that we previously mentioned): patients with increasing body weight or B-type natriuretic peptide levels (proxies of sodium retention) at the end of the enrichment period were excluded to ensure the safety of the intervention [81]. The main methodological difference between the biomarker-based enrichment and adaptive designs is that the previous one ignores whether patients do respond or do not respond to the new experimental treatment. Conversely, in the adaptive enrichment design, a main step is represented by monitoring the treatment effect for a specific biomarker, before randomization. However, a clinical benefit has also been observed in the nonresponder patients enrolled in the SONAR trial [81]. Incidence of primary outcome was slightly lower, even if not significantly, in nonresponder patients assigned to atrasentan compared with placebo. Moreover, nonresponder patients showed a reduction in albuminuria during follow-up. These findings were truly unexpected, since only a small portion of nonresponders showed an albuminuria reduction during the enrichment phase. The phenomenon of regression to the mean played a role in this case, so that nonresponder patients were instead responders during the enrichment. It seems also possible that responders have been insufficiently separated from nonresponders, due to the random variation of proteinuria that could have masked the true response to atrasentan $[81,100]$. Not unlikely is the possibility that a legacy effect may have played a role in the placebo arm; indeed, all patients had been actively treated with atrasentan for 6 weeks before being randomized in placebo and (again) atrasentan. Finally, it has been hypothesized that atrasentan could slow renal disease progression through mechanisms other than change in albuminuria [101].

These issues have raised concerns around the assessment of the individual response to a treatment through the change of biomarker levels (i.e., albuminuria) in large 
intervention studies and increased the interest on how to improve the methodology of enrichment design and how to find other study designs that are able to overcome the individual variability in drug response. To achieve that, we need to move from large international trials to smaller studies that will not encompass all the spectrum of kidney diseases, but only patients with the same type of kidney damage and the same response to treatment.

One strategy is to test 1 or 2 treatments in more than 1 disease within the same trial, the so-called master trial protocol [102]. Master protocols may be planned with multiple interventions, each targeting a specific biomarker-defined population or disease subtype. Under this design, structured platform, umbrella, and basket trials are grouped together (Table 2). Platform trials could be considered a direct extension of enrichment design trials. While the adaptive enrichment studies, like the SONAR, are designed to investigate 1 drug in homogenous patient population, platform trials are planned to identify effective treatments tailored to a particular subgroup of patients [103]. The term "platform" in particular refers to the creation of an experimental platform that will continue to exist after the evaluation of any particular treatment, being generally long-term studies [104]. Within the platform, multiple treatments are started or discontinued. Subgroups from heterogeneous populations could be selected for treatment based on histology, biomarkers, disease risk factors, clinical variables such as age or gender. Next, decision rules (e.g., the likelihood of a treatment benefit) or Bayesian probabilities are used to assess whether a treatment should be continued or discontinued, based on its efficacy. If continued, a treatment could be moved to the clinical setting and could replace or implement the standard of care. Conversely, if a treatment is discontinued, it may be replaced by a new one. This approach allows saving time and resources, since a unique trial infrastructure could be used to evaluate multiple drugs efficiently; to select the best treatment for a subgroup of patients, thus improving the clinical decisionmaking for future individual patients [105]; to avoid unnecessary/unresponsive treatments that last for long time, while the disease progresses [106]. Such interesting frontier of clinical trials has also been recently imported in the nephrology field through the proposal of a global CKD-platform approach [107]. Similar strategies, as those seen for the platform, are used in the "basket" and "umbrella" trials (Table 2). Biomarker-based approaches also need more consideration in future CKD clinical trials. Biomarkers could detect patients who are at increased risk of developing a cardiorenal outcome. For example, several clinical trials, conducted in the previous decades, enrolled CKD patients based on the levels of eGFR or proteinuria. This is also called prognostic enrichment based on biomarkers. Interestingly, biomarkers could also be measured to identify a population which more likely can respond to a particular treatment before exposing the population to such a treatment (predictive enrichment) [71].

Finally, another type of clinical trial design deserves consideration in the context of precision nephrology, as it allows an understanding of how to overcome the drug resistance to standard treatments: the crossover study. In this alternative study design, more treatment periods with different drugs are used, and correlations between responses to 1 drug with each other are measured. The "ROTATE" clinical trial is an example of such a study, in which patients receive, in random order, 4 weeks of the ARB telmisartan, the SGLT2 inhibitor empagliflozin, the DPP4 inhibitor linagliptin, and the glycosaminoglycan sulodexide with 4-week washout periods in between (NCT03504566). This trial is designed to assess individual drug response and will provide additional insight into which patients respond best to which drug. More crossover studies testing interventions with completely different mechanism of actions from RAAS-I should be performed in the future.

Finally, drug development, as well as new clinical trials design, also requires support from the regulatory agencies, European Medicine Agency (EMA) and FDA [108]. It has been advocated that EMA and FDA should shorten the duration of the consultation procedure before giving the "qualification opinion," which is mandatory before starting a clinical trial $[109,110]$. This is important since novel trials, as previously described, could also enroll a small number of patients and could last for short periods of time. Moreover, mechanisms, which can allow for a better collaboration between FDA and EMA (periodic teleconferences, disease-specific meetings, and research consortia), should be implemented in order to reach the precision medicine aims [111].

\section{Conclusions}

In conclusion, precision nephrology is not confined to targeting treatment for each patient but also encompasses individualization of risk, clinical management, and prognosis [112-114]. The variability of CKD, in its broader definition, is an old concept. CKD patients have variable outcomes, which often also take a long time to occur, 
and show variable responses to treatment. Moreover, CKD is per se characterized by a variability in the risk factors which are active in each patient at each stage of the disease. For all these reasons, nephrologists have based clinical decisions mainly on their knowledge and experience. A relevant step forward has been represented by the incorporation of epidemiology in CKD. Precision nephrology adds opportunities and challenges for both observational as well as interventional studies.

In observational studies, personalized information on a patient's prognosis can be reached by building individual risk prediction models. The process of risk prediction model building requires that (1) the research question and the intended use are clearly specified; (2) the population under study and outcomes of interest are well defined; (3) variables are included in the model using a rigorous statistical approach; (4) models provide individual measures, such as calibration, discrimination, and GOF. For the trial perspective, efforts can be made to ameliorate the individualization of treatment. This goal can be attained by implementing new designs such as adaptive enrichment studies, which take into account the individual response to treatment or crossover studies, where patients undergo a sequence of treatments over time. More sophisticated designs like the "umbrella," "basket," and "platform" trials will be tested in the near future.

Therefore, a very important challenge is to better understand variability of CKD and variability of individual drug response. The term variability accompanies a large part of experimental and observational clinical research and reconciles the 2 parts with the common aim that a better comprehension and assessment of variability lead to a better prognosis. A proof of this theory is represented by the inclusion in the modern $\mathrm{CV}$ risk prediction models computing 5- or 10-year CV risk, the information of response (or not response) to treatments together with the standard laboratory and clinical variables. This is done in the U-prevent CV risk calculator [64]. Novel "omics" techniques have demonstrated to be promising in detecting the specific pathopshysiological process in each patient that could be matched with a drug with an appropriate treatment. It would be desirable that in the future, also nephrologists benefit from more and also more specific tools to improve their clinical practice which is needed to guarantee better treatment of patients and, in turn, better clinical outcomes.

\section{Acknowledgements}

We thank all the investigators from the Renal Unit of the Magna Graecia University of Catanzaro (Italy) and "L. Vanvitelli" University of Naples (Italy), and the methodological support from the University Medical Center Groningen (the Netherlands) and Bicocca University of Milan (Italy).

\section{Conflict of Interest Statement}

The authors have no conflicts of interest to declare.

\section{Funding Sources}

The authors received no specific funding for this work.

\section{Author Contributions}

Substantial contributions to the conception or design of the work: M.P., M.A., M.J.P., and L.D.N. Drafting the work or revising it critically for important intellectual content: M.P., M.A., M.J.P., G.C., and L.A. Final approval of the version to be published: M.P., M.A., M.J.P., L.D.N., G.C., L.A., C.G., S.B., and I.G. Agreement to be accountable for all aspects of the work in ensuring that questions related to the accuracy or integrity of any part of the work are appropriately investigated and resolved: M.P., M.A., M.J.P., L.D.N., G.C., L.A., C.G., S.B., and I.G.

\section{References}

Precision Nephrology in Observational and Intervention Studies
1 Levin A, Stevens PE. Summary of KDIGO 2012 CKD Guideline: behind the scenes, need for guidance, and a framework for moving forward. Kidney Int. 2014;85(1):49-61.

2 Collaborators GBDCoD. Global, regional, and national age-sex specific mortality for 264 causes of death, 1980-2016: a systematic analysis for the Global Burden of Disease Study 2016. Lancet. 2017;390(10100):1151-210.

3 Provenzano M, Mancuso C, Garofalo C, De Nicola L, Andreucci M. [Temporal variation of chronic kidney disease's epidemiology]. G Ital Nefrol. 2019;36(2):2019.
4 Russo D, Morrone LF, Errichiello C, De Gregorio MG, Imbriaco M, Battaglia Y, et al. Impact of BMI on cardiovascular events, renal function, and coronary artery calcification. Blood Purif. 2014;38(1):1-6.

5 De Nicola L, Provenzano M, Chiodini P, D’Arrigo G, Tripepi G, Del Vecchio L, et al. Prognostic role of LDL cholesterol in non-dialysis chronic kidney disease: multicenter prospective study in Italy. Nutr Metab Cardiovasc Dis. 2015;25(8):756-62. 
6 De Nicola L, Provenzano M, Chiodini P, Borrelli S, Russo L, Bellasi A, et al. Epidemiology of low-proteinuric chronic kidney disease in renal clinics. PLoS One. 2017 Feb 17;12(2): e0172241.

7 Perco P, Pena M, Heerspink HJL, Mayer G. Multimarker panels in diabetic kidney disease: the way to improved clinical trial design and clinical practice?. Kidney Int Rep. 2018 Dec;4(2):212-21.

8 Lambers Heerspink HJ, Weldegiorgis M, Inker LA, Gansevoort R, Parving HH, Dwyer JP, et al. Estimated GFR decline as a surrogate end point for kidney failure: a post hoc analysis from the reduction of end points in noninsulin-dependent diabetes with the Angiotensin II Antagonist Losartan (RENAAL) study and Irbesartan Diabetic Nephropathy Trial (IDNT). Am J Kidney Dis. 2014;63(2): 244-50.

9 Moranne O, Froissart M, Rossert J, Gauci C, Boffa JJ, Haymann JP, et al. Timing of onset of CKD-related metabolic complications. J Am Soc Nephrol. 2009;20(1):164-71.

10 De Nicola L, Chiodini P, Zoccali C, Borrelli S, Cianciaruso B, Di Iorio B, et al. Prognosis of CKD patients receiving outpatient nephrology care in Italy. Clin J Am Soc Nephrol. 2011; 6(10):2421-8.

11 Rothman K, Greenland S, Lash TL. Modern epidemiology, 3rd ed. Philadelphia, PA: Lippincott Williams \& Wilkins; 2008.

12 Levey AS, Astor BC, Stevens LA, Coresh J. Chronic kidney disease, diabetes, and hypertension: what's in a name? Kidney Int. 2012; 78(1):19-22.

13 Adler AI, Stevens RJ, Manley SE, Bilous RW, Cull CA, Holman RR; UKPDS Group. Development and progression of nephropathy in type 2 diabetes: the United Kingdom Prospective Diabetes Study (UKPDS 64). Kidney Int. 2003 Jan;63(1):225-32.

14 Pena MJ, Stenvinkel P, Kretzler M, Adu D, Agarwal SK, Coresh J, et al. Strategies to improve monitoring disease progression, assessing cardiovascular risk, and defining prognostic biomarkers in chronic kidney disease. Kidney Int. 2017 Oct;7(2):107-13.

15 de Vries JK, Levin A, Loud F, Adler A, Mayer $G$, Pena $M J$. Implementing personalized medicine in diabetic kidney disease: stakeholders' perspectives. Diabetes Obes Metab. 2018;20(Suppl 3):24-9.

16 Collins FS, Varmus H. A new initiative on precision medicine. N Engl J Med. 2015; 372(9):793-5.

17 Astor BC, Matsushita K, Gansevoort RT, van der Velde M, Woodward M, Levey AS, et al. Lower estimated glomerular filtration rate and higher albuminuria are associated with mortality and end-stage renal disease. A collaborative meta-analysis of kidney disease population cohorts. Kidney Int. 2011;79(12): 1331-40.

18 Gansevoort RT, Matsushita K, van der Velde M, Astor BC, Woodward M, Levey AS, et al. Lower estimated GFR and higher albumin- uria are associated with adverse kidney outcomes. A collaborative meta-analysis of general and high-risk population cohorts. Kidney Int. 2011;80(1):93-104.

19 Borrelli S, Garofalo C, Mallamaci F, Tripepi G, Stanzione G, Provenzano M, et al. Shortterm blood pressure variability in nondialysis chronic kidney disease patients: correlates and prognostic role on the progression of renal disease. J Hypertens. 2018;36(12):2398405.

20 Janes H, Pepe MS, Gu W. Assessing the value of risk predictions by using risk stratification tables. Ann Intern Med. 2008;149(10):75160.

21 Kidney Disease Improving Global Outcomes (KDIGO) CKD Work Group. KDIGO 2012 clinical practice guideline for the evaluation and management of chronic kidney disease. Kidney Int Suppl. 2013;3:31-150.

22 Hall YN, Himmelfarb J. The CKD classification system in the precision medicine era. Clin J Am Soc Nephrol. 2017;12(2):346-8.

23 Deckert T, Feldt-Rasmussen B, Borch-Johnsen K, Jensen T, Kofoed-Enevoldsen A. Albuminuria reflects widespread vascular damage. The Steno hypothesis. Diabetologia 1989; 32(4):219-26

24 De Nicola L, Gabbai FB, Liberti ME, Sagliocca A, Conte G, Minutolo R. Sodium/glucose cotransporter 2 inhibitors and prevention of diabetic nephropathy: targeting the renal tubule in diabetes. Am J Kidney Dis. 2014;64(1):16-24.

25 De Nicola L, Provenzano M, Chiodini P, Borrelli S, Garofalo C, Pacilio M, et al. Independent role of underlying kidney disease on renal prognosis of patients with chronic kidney disease under nephrology care. PLoS One. 2015 May 20;10(5):e0127071.

26 Haynes R, Staplin N, Emberson J, Herrington WG, Tomson C, Agodoa L, et al. Evaluating the contribution of the cause of kidney disease to prognosis in CKD: results from the Study of Heart and Renal Protection (SHARP). Am J Kidney Dis. 2014 Jul;64(1):40-8.

27 Tangri N, Stevens LA, Griffith J, Tighiouart $\mathrm{H}$, Djurdjev O, Naimark D, et al. A predictive model for progression of chronic kidney disease to kidney failure. JAMA. 2011 Apr 20; 305(15):1553-9.

28 Kannel WB, Larson M. Long-term epidemiologic prediction of coronary disease. The Framingham experience. Cardiology. 1993; $82(2-3): 137-52$.

29 Keane WF, Zhang Z, Lyle PA, Cooper ME, de Zeeuw D, Grunfeld JP, et al. Risk scores for predicting outcomes in patients with type 2 diabetes and nephropathy: the RENAAL study. Clin J Am Soc Nephrol. 2006;1(4):7617

30 Tangri N, Grams ME, Levey AS, Coresh J, Appel LJ, Astor BC, et al. Multinational assessment of accuracy of equations for predicting risk of kidney failure: a meta-analysis. JAMA. 2016;315(2):164-74.

31 Schroeder EB, Yang X, Thorp ML, Arnold BM, Tabano DC, Petrik AF, et al. Predicting 5-year risk of RRT in stage 3 or 4 CKD: development and external validation. Clin J Am Soc Nephrol. 2017;12(1):87-94.

32 Grams ME, Sang Y, Ballew SH, Carrero JJ, Djurdjev O, Heerspink HJL, et al. Predicting timing of clinical outcomes in patients with chronic kidney disease and severely decreased glomerular filtration rate. Kidney Int. 2018; 93(6): 1442-51.

33 Evans M, Grams ME, Sang Y, Astor BC, Blankestijn PJ, Brunskill NJ, et al. Risk factors for prognosis in patients with severely decreased GFR. Kidney Int Rep. 2018 Jan 11; 3(3):625-37.

34 Eckardt KU, Bansal N, Coresh J, Evans M, Grams ME, Herzog CA, et al. Improving the prognosis of patients with severely decreased glomerular filtration rate (CKD G4+): conclusions from a kidney disease: Improving Global Outcomes (KDIGO) Controversies Conference. Kidney Int. 2018;93(6):1281-92.

35 Taal MW, Brenner BM. Predicting initiation and progression of chronic kidney disease: developing renal risk scores. Kidney Int. 2006 Nov;70(10):1694-705.

36 Kadatz MJ, Lee ES, Levin A. Predicting progression in CKD: perspectives and precautions. Am J Kidney Dis. 2016;67(5):779-86.

37 Pepe MS, Kerr KF, Longton G, Wang Z. Testing for improvement in prediction model performance. Stat Med. 2013;32(9):1467-82.

38 Tangri N, Kitsios GD, Inker LA, Griffith J, Naimark DM, Walker S, et al. Risk prediction models for patients with chronic kidney disease: a systematic review. Ann Intern Med. 2013;158(8):596-603.

39 Lash JP, Go AS, Appel LJ, He J, Ojo A, Rahman M, et al. Chronic Renal Insufficiency Cohort (CRIC) study: baseline characteristics and associations with kidney function. Clin J Am Soc Nephrol. 2009 Aug;4(8):1302-11.

40 Provenzano M, Chiodini P, Minutolo R, Zoccali C, Bellizzi V, Conte G, et al. Reclassification of chronic kidney disease patients for end-stage renal disease risk by proteinuria indexed to estimated glomerular filtration rate: multicentre prospective study in nephrology clinics. Nephrol Dial Transpl. 2018 Jul 23; 35(1):138-47.

41 De Nicola L, Donfrancesco C, Minutolo R, Lo Noce C, Palmieri L, De Curtis A, et al. Prevalence and cardiovascular risk profile of chronic kidney disease in Italy: results of the 200812 National Health Examination Survey. Nephrol Dial Transplant. 2015 May;30(5): 806-14.

42 Minutolo R, Gabbai FB, Provenzano M, Chiodini P, Borrelli S, Garofalo C, et al. Cardiorenal prognosis by residual proteinuria level in diabetic chronic kidney disease: pooled analysis of four cohort studies. Nephrol Dial Transplant. 2018 Nov 1;33(11):1942-9.

43 Turin TC, Coresh J, Tonelli M, Stevens PE, de Jong PE, Farmer CK, et al. Short-term change in kidney function and risk of end-stage renal disease. Nephrol Dial Transplant. 2012 Oct; 27(10):3835-43. 
44 Brück K, Jager KJ, Zoccali C, Bello AK, Minutolo R, Ioannou K, et al. Different rates of progression and mortality in patients with chronic kidney disease at outpatient nephrology clinics across Europe. Kidney Int. 2018 Jun; 93(6):1432-41.

45 Borrelli S, Leonardis D, Minutolo R, Chiodini P, De Nicola L, Esposito C, et al. Epidemiology of CKD regression in patients under nephrology care. PLoS One. 2015 Oct 13;10(10): e0140138.

46 Li L, Astor BC, Lewis J, Hu B, Appel LJ, Lipkowitz MS, et al. Longitudinal progression trajectory of GFR among patients with CKD. Am J Kidney Dis. 2012;59(4):504-12,

47 O'Hare AM, Batten A, Burrows NR, Pavkov ME, Taylor L, Gupta I, et al. Trajectories of kidney function decline in the 2 years before initiation of long-term dialysis. Am J Kidney Dis. 2012;59(4):513-22.

48 Lewis EJ, Hunsicker LG, Clarke WR, Berl T, Pohl MA, Lewis JB, et al. Renoprotective effect of the angiotensin-receptor antagonist irbesartan in patients with nephropathy due to type 2 diabetes. N Engl J Med. 2001;345(12): 851-60.

49 Verschuren WM, Jacobs DR, Bloemberg BP, Kromhout D, Menotti A, Aravanis C, et al. Serum total cholesterol and long-term coronary heart disease mortality in different cultures. Twenty-five-year follow-up of the seven countries study. JAMA. 1995;274(2):1316.

50 De Nicola L, Locatelli F, Conte G, Minutolo R. Responsiveness to erythropoiesis-stimulating agents in chronic kidney disease: does geography matter? Drugs. 2014;74(2):159-68.

51 Penno G, Solini A, Bonora E, Fondelli C, Orsi E, Zerbini G, et al. Clinical significance of nonalbuminuric renal impairment in type 2 diabetes. J Hypertens. 2011;29(9):1802-9.

52 Iseki K. Gender differences in chronic kidney disease. Kidney Int. 2008;74(4):415-7.

53 Minutolo R, Gabbai FB, Chiodini P, Provenzano M, Borrelli S, Garofalo C, et al. Sex differences in the progression of CKD among older patients: pooled analysis of 4 cohort studies. Am J Kidney Dis. 2020;75(1):30-8.

54 Pearce N. Analytical implications of epidemiological concepts of interaction. Int J Epidemiol. 1989 Dec;18(4):976-80.

55 Hallan SI, Rifkin DE, Potok OA, Katz R, Langlo KA, Bansal N, et al. Implementing the European Renal Best Practice Guidelines suggests that prediction equations work well to differentiate risk of end-stage renal disease vs. death in older patients with low estimated glomerular filtration rate. Kidney Int. 2019 Sep;96(3):728-37.

56 Weiner DE, Tighiouart H, Elsayed EF, Griffith JL, Salem DN, Levey AS, et al. The Framingham predictive instrument in chronic kidney disease. J Am Coll Cardiol. 2007;50(3):21724.

57 Lerner B, Desrochers S, Tangri N. Risk prediction models in CKD. Semin Nephrol. 2017 Mar;37(2):144-50.
58 Esposito P, Estienne L, Serpieri N, Ronchi D, Comi GP, Moggio M, et al. Rhabdomyolysisassociated acute kidney injury. Am J Kidney Dis. 2018;71(6):A12-4.

59 Roy J, Shou H, Xie D, Hsu JY, Yang W, Anderson $\mathrm{AH}$, et al. Statistical methods for cohort studies of CKD: prediction modeling. Clin J Am Soc Nephrol. 2017 Jun 7;12(6): 1010-7.

60 Nagelkerke NJD. A note on a general definition of the coefficient of determination. Biometrika. 1991;78(3):691-2.

61 Steyerberg EW, Vickers AJ, Cook NR, Gerds T, Gonen M, Obuchowski N, et al. Assessing the performance of prediction models: a framework for traditional and novel measures. Epidemiology. 2010 Jan;21(1):128-38.

62 Han D, Kolli KK, Gransar H, Lee JH, Choi S-Y, Chun EJ, et al. Machine learning based risk prediction model for asymptomatic individuals who underwent coronary artery calcium score: comparison with traditional risk prediction approaches [published online ahead of print, 2019 Sep 23]. J Cardiovasc Comput Tomogr. 2019;S1934-5925(19):30255-2.

63 Cooney MT, Selmer R, Lindman A, Tverdal A, Menotti A, Thomsen T, et al. Cardiovascular risk estimation in older persons: SCORE O.P. Eur J Prev Cardiol. 2016;23(10):1093103.

64 Rossello X, Dorresteijn JA, Janssen A, Lambrinou E, Scherrenberg M, Bonnefoy-Cudraz $\mathrm{E}$, et al. Risk prediction tools in cardiovascular disease prevention: a report from the ESC Prevention of CVD Programme led by the European Association of Preventive Cardiology (EAPC) in collaboration with the Acute Cardiovascular Care Association (ACCA) and the Association of Cardiovascular Nursing and Allied Professions (ACNAP). Eur Heart J Acute Cardiovasc Care. 2019; 2048872619858285.

65 Levin A, Tonelli M, Bonventre J, Coresh J, Donner JA, Fogo AB, et al. Global kidney health 2017 and beyond: a roadmap for closing gaps in care, research, and policy. Lancet. 2017;390(10105):1888-917.

66 Mead N, Bower P. Patient-centredness: a conceptual framework and review of the empirical literature. Soc Sci Med. 2000;51(7):1087110.

67 Ballew SH, Matsushita K. Cardiovascular risk prediction in CKD. Semin Nephrol. 2018; 38(3):208-16

68 Peralta CA, Shlipak MG, Judd S, Cushman M, McClellan W, Zakai NA, et al. Detection of chronic kidney disease with creatinine, cystatin $\mathrm{C}$, and urine albumin-to-creatinine ratio and association with progression to end-stage renal disease and mortality. JAMA. 2011; 305(15):1545-52.

69 Isakova T. Fibroblast growth factor 23 and adverse clinical outcomes in chronic kidney disease. Curr Opin Nephrol Hypertens. 2012; 21(3):334-40.

70 Critselis E, Lambers Heerspink H. Utility of the CKD273 peptide classifier in predicting chronic kidney disease progression. Nephrol Dial Transp. 2016;31(2):249-54.

71 Sun L, Zou LX, Chen MJ. Make precision medicine work for chronic kidney disease. Med Princ Pract. 2017;26(2):101-7.

72 Good DM, Zürbig P, Argilés A, Bauer HW, Behrens G, Coon JJ, et al. Naturally occurring human urinary peptides for use in diagnosis of chronic kidney disease. Mol Cell Proteomics. 2010;9(11):2424-37.

73 Argilés Á, Siwy J, Duranton F, Gayrard N, Dakna M, Lundin U, et al. CKD273, a new proteomics classifier assessing CKD and its prognosis. PLoS One. 2013;8(5):e62837.

74 Schanstra JP. Diagnosis and prediction of progression of chronic kidney disease by assessment of urinary peptides. J Am Soc Nephrol. 2015;26(8):1999-2010.

75 Roscioni SS, de Zeeuw D, Hellemons ME, Mischak H, Zürbig P, Bakker SJ, et al. A urinary peptide biomarker set predicts worsening of albuminuria in type 2 diabetes mellitus. Diabetologia. 2013;56(2):259-67.

76 Brenner BM, Cooper ME, de Zeeuw D, Keane WF, Mitch WE, Parving HH, et al. Effects of losartan on renal and cardiovascular outcomes in patients with type 2 diabetes and nephropathy. N Engl J Med. 2001;345(12):8619.

77 Appel LJ, Wright JTJr, Greene T, Agodoa LY, Astor BC, Bakris GL, et al. Intensive bloodpressure control in hypertensive chronic kidney disease. N Engl J Med. 2010 Sep 2;363(10): 918-29.

78 Cheung AK, Rahman M, Reboussin DM, Craven TE, Greene T, Kimmel PL, et al. Effects of intensive BP control in CKD. J Am Soc Nephrol. 2017;28(9):2812-23.

79 de Zeeuw D, Remuzzi G, Parving HH, Keane WF, Zhang Z, Shahinfar S, et al. Proteinuria, a target for renoprotection in patients with type 2 diabetic nephropathy: lessons from RENAAL. Kidney Int. 2004;65(6):2309-20.

80 The GISEN Group (Gruppo Italiano Studi Epidemiologici in Nefrologia). Randomized placebo-controlled trial of effect of ramipril on decline in glomerular filtration rate and progression to terminal renal failure in proteinuric, non-diabetic nephropathy. Lancet. 1997;349:1857-63.

81 Heerspink HJL, Parving HH, Andress DL, Bakris G, Correa-Rotter R, Hou FF, et al. Atrasentan and renal events in patients with type 2 diabetes and chronic kidney disease (SONAR): a double-blind, randomised, placebo-controlled trial. Lancet. 2019 May 11; 393(10184):1937-47.

82 Perkovic V, Jardine MJ, Neal B, Bompoint S, Heerspink HJL, Charytan DM, et al. Canagliflozin and renal outcomes in type 2 diabetes and nephropathy. N Engl J Med. 2019; 380(24):2295-306.

83 Pfeffer MA, Burdmann EA, Chen CY, Cooper ME, de Zeeuw D, Eckardt KU, et al. A trial of darbepoetin alfa in type 2 diabetes and chronic kidney disease. N Engl J Med. 2009 Nov 19; 361(21):2019-32.
Precision Nephrology in Observational and Intervention Studies
Nephron 2020;144:463-478 DOI: $10.1159 / 000508983$ 
84 Tonelli M, Muntner P, Lloyd A, Manns BJ, Klarenbach S, Pannu N, et al. Risk of coronary events in people with chronic kidney disease compared with those with diabetes: a population-level cohort study. Lancet. 2012; 380(9844):807-14.

85 Atkins RC, Briganti EM, Lewis JB, Hunsicker LG, Braden G, Champion de Crespigny PJ, et al. Proteinuria reduction and progression to renal failure in patients with type 2 diabetes mellitus and overt nephropathy. Am J Kidney Dis. 2005;45(2):281-7.

86 Heerspink HJ, Ninomiya T, Persson F, Brenner BM, Brunel P, Chaturvedi N, et al. Is a reduction in albuminuria associated with renal and cardiovascular protection? A post hoc analysis of the ALTITUDE trial. Diabetes Obes Metab. 2016;18(2):169-77.

87 Petrykiv SI, Laverman GD, de Zeeuw D, Heerspink HJL. The albuminuria-lowering response to dapagliflozin is variable and reproducible among individual patients. Diabetes Obes Metab. 2017;19(10):1363-70.

88 Petrykiv SI, Laverman GD, Persson F, Vogt L, Rossing P, de Borst $\mathrm{MH}$, et al. Pooled analysis of multiple crossover trials to optimize individual therapy response to renin-angiotensin-aldosterone system intervention. Clin J Am Soc Nephrol. 2017;12(11):1804-13.

89 Schievink B, de Zeeuw D, Parving HH, Rossing P, Lambers Heerspink HJ The renal protective effect of angiotensin receptor blockers depends on intra-individual response variation in multiple risk markers. Br J Clin Pharmacol. 2015;80(4):678-86.

90 Heerspink HJ, de Zeeuw D. The kidney in type 2 diabetes therapy. Rev Diabet Stud. 2011;8(3):392-402.

91 Provenzano M, Rivoli L, Garofalo C, Faga T, Pelagi E, Perticone M, et al. Renal resistive index in chronic kidney disease patients: possible determinants and risk profile. PLoS One. 2020 Apr;15(4):e0230020.

92 Trudu M, Janas S, Lanzani C, Debaix H, Schaeffer C, Ikehata M, et al. Common noncoding UMOD gene variants induce salt-sensitive hypertension and kidney damage by increasing uromodulin expression. Nat Med. 2013;19(12):1655-60.
93 Young GH, Wu VC. Klotho methylation is linked to uremic toxins and chronic kidney disease. Kidney Int. 2012;81(7):611-2.

94 Carew RM, Wang B, Kantharidis P. The role of EMT in renal fibrosis. Cell Tissue Res. 2012;347(1):103-16.

95 Lee SY, Kim SI, Choi ME. Therapeutic targets for treating fibrotic kidney diseases. Transl Res. 2014;165(4):512-30.

96 Heerspink HJ, Grobbee DE, de Zeeuw D. A novel approach for establishing cardiovascular drug efficacy. Nat Rev Drug Discov. 2014;13(12):942.

97 Mayer G, Heerspink HJ, Aschauer C, Heinzel A, Heinze G, Kainz A, et al. Systems biology-derived biomarkers to predict progression of renal function decline in type 2 diabetes. Diabetes Care. 2017;40(3):391-7.

98 Lindhardt M, Persson F, Currie G, Pontillo C, Beige J, Delles C, et al. Proteomic prediction and Renin angiotensin aldosterone system Inhibition prevention Of early diabetic nephRopathy in TYpe 2 diabetic patients with normoalbuminuria (PRIORITY): essential study design and rationale of a randomised clinical multicentre trial. BM] Open. 2016 Mar 2;6(3):e010310.

99 Tofte N, Lindhardt M, Adamova K, Bakker SJL, Beige J, Beulens JWJ, et al. Early detection of diabetic kidney disease by urinary proteomics and subsequent intervention with spironolactone to delay progression (PRIORITY): a prospective observational study and embedded randomised placebocontrolled trial. Lancet Diabetes Endocrinol. 2020;8(4):301-12.

100 Provenzano M, Garofalo C, Chiodini P, Mancuso C, Barbato E, De Nicola L, et al. Role of proteinuria in clinical research: for each old-answer, a new key-question. Recenti Prog Med. 2020;111(2):74-81.

101 Pena MJ, de Zeeuw D, Andress D, Brennan JJ, Correa-Rotter R, Coll B, et al. The effects of atrasentan on urinary metabolites in patients with type 2 diabetes and nephropathy. Diabetes Obes Metab. 2017;19(5):749-53.

102 Woodcock J, LaVange LM. Master protocols to study multiple therapies, multiple diseases, or both. N Engl J Med. 2017;377(1):62-70.
103 Berry SM, Connor JT, Lewis RJ. The platform trial: an efficient strategy for evaluating multiple treatments. JAMA. 2015;313(16): 1619-20.

104 Mullard A. Multicompany trials adapt to disciplines beyond cancer. Nat Med. 2014; 20(1):3.

105 Heerspink HJL, List J, Perkovic V. New clinical trial designs for establishing drug efficacy and safety in a precision medicine era. Diabetes Obes Metab. 2018;20(Suppl 3):14-8.

106 Mahon J, Laupacis A, Donner A, Wood T. Randomised study of $\mathrm{n}$ of 1 trials versus standard practice. BMJ. 1996 Apr 27; 312(7038):1069-74

107 de Zeeuw D, Heerspink HJL, Jardine M, Perkovic V Renal trials in diabetes need a platform: time for a global approach? Lancet Diabetes Endocrinol. 2018;6:356-8.

108 Mol PGM, Thompson A, Heerspink HJL Leufkens HGM. Precision medicine in diabetes and diabetic kidney disease: regulatory considerations. Diabetes Obes Metab. 2018; 20(Suppl 3):19-23.

109 EMA. Qualification of novel methodologies for drug development: guidance to applicants (EMA/CHMP/SAWP/72894/2008).

110 Food and Drug Administration. 21st century cures act: qualification of drug development tools. 2020

111 Salgado R, Moore H, Martens JWM, Lively T, Malik S, McDermott U, et al. Societal challenges of precision medicine: bringing order to chaos. Eur J Cancer. 2017;84:325-34.

112 Provenzano M, Coppolino G, Faga T, Garofalo C, Serra R, Andreucci M. Epidemiology of cardiovascular risk in chronic kidney disease patients: the real silent killer. Rev Cardiovasc Med. 2019;20(4):209-20.

113 Provenzano M, Coppolino G, De Nicola L, Serra R, Garofalo C, Andreucci M, et al. Unraveling cardiovascular risk in renal patients: a new take on old tale. Front Cell Dev Biol. 2019 Dec 3;7:314.

114 Galassi A, Brancaccio D, Andreucci VE. Risk for chronic kidney disease in the general population Italian reports for world kidney days 2007-2009. J Nephrol. 2010;23(6):743-6. 